\title{
Los estudios de posgrado en contextos de evaluación. Aproximaciones comparadas entre Argentina, Brasil y Paraguay
}

\author{
Nora Zoila Lamfri \& Sonia Marcela Araujo
}

Resumen:

En el marco de un mercado internacional vigoroso, la oferta de posgrados en América Latina ha tenido un crecimiento sostenido en las últimas tres décadas. Esta expansión ha sido acompañada por el diseño de políticas y marcos legales orientados por los discursos de la calidad de la Educación Superior que otorgaron a la evaluación una fuerza regulatoria estratégica, redefiniendo la relación entre el Estado y las universidades. Particularmente en Argentina, Brasil y Paraguay, la creciente importancia y complejidad de los procesos e impactos producidos por esta expansión amerita un análisis comparado en profundidad que combine elementos estructurales y coyunturales de cada país con tendencias globales de internacionalización y privatización. En este artículo se presentan resultados alcanzados por una Red de investigadores de cuatro universidades públicas: dos de Argentina, una de Brasil y una en el Paraguay, cuyo principal objetivo fue mapear y comparar la situación actual de los posgrados en los tres países. El trabajo colaborativo realizado por la Red se tradujo en una matriz comparativa cuyas dimensiones fueron definidas colectivamente durante el desarrollo de la investigación: génesis y desarrollo, marco regulatorio, oferta, evaluación y acreditación, financiamiento e internacionalización de los posgrados. El estudio comparado permitió advertir tendencias comunes y algunas disimilitudes que devienen de las diferencias en los orígenes, culturas y tradiciones universitarias en cada país y los modos en que la regulación fue procesada por los actores locales.

Palabras clave:

posgrado; estudio comparado; trabajo en red. 


\title{
Os estudos de pós-graduação em contextos de avaliação. Aproximações comparadas entre Argentina, Brasil e Paraguai
}

\begin{abstract}
Resumo: No âmbito de um vigoroso mercado internacional, a oferta de pós-graduação na América Latina teve um crescimento sustentado nas últimas três décadas. Esta expansão foi acompanhada pelo projeto de políticas e por marcos legais guiados pelos discursos da qualidade do Ensino Superior que deram à avaliação uma força reguladora estratégica, redefinindo a relação entre o Estado e as universidades. Particularmente na Argentina, no Brasil e no Paraguai, a crescente importância e complexidade dos processos e impactos produzidos por esta expansão merecem uma profunda análise comparativa que combina elementos estruturais e contextuais de cada país com tendências globais de internacionalização e privatização. Este artigo apresenta os resultados alcançados por uma Rede de pesquisadores de quatro universidades públicas, duas na Argentina, uma no Brasil e outra no Paraguai, cujo principal objetivo foi mapear e comparar a situação atual da pós-graduação nos três países. O trabalho colaborativo realizado pela Rede foi traduzido em uma matriz comparativa cujas dimensões foram definidas coletivamente durante o desenvolvimento da pesquisa: gênese e desenvolvimento, quadro regulatório, oferta, avaliação e credenciamento, financiamento e internacionalização de programas de pós-graduação. O estudo comparativo permitiu notar tendências comuns e algumas desigualdades que resultam das diferenças nas origens, culturas e tradições universitárias em cada país e as formas como o regulamento foi processado pelos atores locais.
\end{abstract}

Palavras-chave: pós-graduação; estudo comparativo; trabalho em rede.

\section{Les études supérieures dans le contexte de l'évaluation. Des approximations comparatives parmi Argentine, le Brésil et le Paraguay}

Resumé: Dans le cadre d'un marché international vigoureux, l'offre d'études supérieures en Amérique Latine a connu une croissance soutenue au cours des trois dernières décennies. Cette expansion s'est accompagnée de la conception de politiques publiques et de cadres juridiques guidés par les discours sur la qualité de l'enseignement supérieur qui ont donné à l'évaluation une force de régulation stratégique, redéfinissant la relation entre l'État et les universités. Particulièrement, en Argentine, au Brésil et au Paraguay, l'importance et la complexité croissantes des processus et impacts produits par cette expansion méritent une analyse comparative approfondie qui combine les éléments structurels et contextuels de chaque pays avec les tendances globales de l'internationalisation et de la privatisation. Cet article présente les résultats obtenus par un réseau de chercheurs de quatre universités publiques, deux en Argentine, une au Brésil et une au Paraguay, dont l'objectif principal était de cartographier et de comparer la situation actuelle du troisième cycle au trois pays. Le travail collaboratif réalisé par le Réseau s'est traduit par une matrice comparative dont les dimensions ont été définies collectivement lors du développement de la recherche: genèse et développement, cadre réglementaire, offre, évaluation et accréditation, financement et internationalisation des programmes d'études supérieures. L'étude comparative a permis de constater des tendances communes et des dissemblances dues aux différences d'origines, de cultures et de traditions universitaires de chaque pays et à la façon dont la réglementation a été traitée par les acteurs locaux.

Mots clés: Diplomé; étude comparative; mise en réseau.

\section{Post-graduate education in the evaluation context. Comparative perspective among Argentina, Brazil and Paraguay}

\begin{abstract}
Within the framework of a vigorous international market, postgraduate education in Latin America has had a sustained growth in the last two decades. This expansion has been accompanied by the design of policies and legal frameworks guided by the discourses of the quality of Higher Education that gave the evaluation a strategic regulatory force, redefining the relationship between the State and the universities. Particularly in Argentina, Brazil, and Paraguay, the growing importance and complexity of the processes and impacts produced by this expansion merits an in depth comparative analysis that combines structural and contextual elements of each country with global trends of internationalization and privatization. This article presents results achieved by a Network of researchers from four public universities, two in Argentina, one in Brazil and another one in Paraguay. Its main objective was to map and compare the current situation of postgraduate education in these countries. The collaborative work carried out by the Network was translated into a comparative matrix whose dimensions were collectively defined during the development of the research: origins and development, regulatory framework, supply, evaluation and accreditation, financing and internationalization of postgraduate programs. The comparative study allowed noticing common tendencies and some dissimilarities that come from the differences in the origins, cultures and university traditions in each country and the ways in which the regulation was processed by the local actors.
\end{abstract}

Keywords: postgraduate education; comparative study; network research. 


\section{Introducción}

A partir de la década de 1990, en el marco de gobiernos de corte neoliberal, se alteró el patrón tradicional de relación de la educación superior con el Estado y la sociedad en los países de la región latinoamericana. Ya sea que se aluda a transformación, crisis, reestructuración, o reforma para referirse a las mutaciones de este nivel educativo, existe acuerdo en que la presencia de una serie de cambios globales en las esferas política, económica, social y cultural sostuvo dichas mutaciones. En efecto, el avance del proceso de globalización, el papel de la producción de conocimiento en el desarrollo económico y la inserción competitiva de las naciones, así como la revolución de la información y de las comunicaciones, juntamente con la crisis fiscal y el aumento de la demanda de educación superior provocaron la erosión de los modelos y patrones tradicionales de universidad. La exigencia de nuevas respuestas comenzó a desmantelar la cultura sostenida en valores tradicionalmente arraigados en las instituciones y en quienes ejercen la profesión académica: autonomía, libertad de cátedra, búsqueda del conocimiento como un fin en sí mismo y el valor de la educación como un bien público y un derecho universal, concepción que fue reemplazada por la de un bien sujeto a las reglas del mercado.

En el marco de las políticas de promoción de la calidad educativa, la evaluación se instaló como estrategia de gestión con múltiples referentes: instituciones, docentes, investigadores, carreras de grado y posgrado, currículos. En los distintos países fueron creadas agencias con funciones específicas para la evaluación de la calidad, asentadas en postulados generales compartidos, aunque con diferencias en los aspectos instrumentales. A estas disimilitudes se agrega, además, que la instalación de los sistemas de evaluación se llevó a cabo en países con sistemas universitarios diferentes en sus orígenes y tradiciones culturales y educativas.

En un contexto caracterizado por la competitividad internacional en el que el conocimiento juega un rol fundamental, el nivel cuaternario cobra significación por cuanto es de esperar sea un ámbito con capacidad para la creación, transmisión y transferencia de conocimientos y competencias actualizados en diversas poblaciones académicas y profesionales. Sin embargo, también es cierto que los sistemas de posgrado tienen una evolución particular y cumplen diversas funciones en los contextos nacionales en los que se insertan, razón por la cual es de fundamental importancia indagar estos aspectos así como los efectos de los sistemas de evaluación creados para su regulación.

El artículo presenta resultados alcanzados por una Red de Investigadores de cuatro universidades públicas de tres países: La Universidad Nacional del Centro de la Provincia de Buenos Aires (Argentina), la Universidad Estadual de Campinas (Brasil), la Universidad Nacional de Asunción (Paraguay) y la Universidad Nacional de Córdoba (Argentina), cuyo principal propósito fue mapear y comparar la situación actual de los posgrados en Argentina, Brasil y Paraguay. El trabajo se realizó y financió como parte de una iniciativa 
del Núcleo de Estudios e Investigaciones sobre Educación Superior del MERCOSUR (NEIES) del Sector Educativo del MERCOSUR (SEM), coordinado por el Programa de Internacionalización de la Educación Superior y Cooperación Internacional (PIESCI) del Ministerio de Educación de Argentina.

La exposición se estructura en cuatro apartados: el primero presenta los objetivos generales del trabajo, el segundo ofrece precisiones sobre el encuadre teórico metodológico asumido para la comparación y el funcionamiento de la Red, el tercero sintetiza una selección de los principales hallazgos y el último incluye reflexiones finales en torno a la investigación realizada.

\section{Los objetivos del estudio}

La investigación estuvo orientada a analizar las principales características que ha asumido el desarrollo de los posgrados en Argentina, Brasil y Paraguay en las últimas tres décadas desde una perspectiva comparada, identificando convergencias y divergencias en las tendencias y procesos de cambio en las culturas de producción de conocimientos, en su vínculo con políticas de evaluación y regulación estatal. Como propósito adicional la Red trató de aportar conocimientos para el fortalecimiento del proceso de formulación de políticas públicas de posgrado y sus articulaciones con otras problemáticas relacionadas de la educación superior, con la intención de contribuir a la toma de decisiones en los ámbitos institucionales y gubernamentales para el logro de acciones colaborativas y de integración entre los posgrados de los diferentes países.

En este sentido, las indagaciones se propusieron identificar etapas en el desarrollo histórico de los posgrados en Argentina, Brasil y Paraguay; caracterizar la oferta de posgrados en los tres países en el contexto de los cambios en las relaciones sociedad, estado y educación superior en el último cuarto del siglo XX; caracterizar los mecanismos de regulación de los posgrados en cada contexto nacional para su posterior análisis comparativo; analizar los sistemas de evaluación y acreditación de los posgrados con vistas a reconocer los impactos en las carreras y en las instituciones; y caracterizar los mecanismos de financiamiento establecidos para su sostenimiento así como las formas que han ido asumiendo los procesos de internacionalización de los posgrados en los países estudiados.

\section{Diseño teórico metodológico de la investigación}

Para el abordaje comparado, la idea nodal de "dilema institucional" orienta un análisis histórico comparativo que permite identificar culturas sistémicas e institucionales nuevas, en el sentido de poner en foco nuevos elementos que devienen del impacto de la evaluación como regulación de los posgrados (Aguilar, 2011). Se presupone la existencia 
de culturas de producción de conocimientos configurados históricamente en los tres países, por lo que se incorpora también la noción de "punto de partida y ritmo" que en su desarrollo analítico permite estudiar las "semejanzas y diferencias cruciales" que dan consistencia metodológica y validez al ejercicio comparativo (Aguilar, 2016). Estas definiciones fueron puestas en diálogo con los datos relevados en diversas fuentes bibliográficas y fuentes primarias como documentos, normativas y estadísticas oficiales; y con diferentes herramientas según los requerimientos a través de encuestas y entrevistas a informantes clave. Resulta relevante mencionar que si bien algunos países han avanzado en bases de datos muy consolidadas y accesibles, en otros todavía hay escasas posibilidades de contar con la información requerida por lo que la disponibilidad y acceso a los mismos es una cuestión sensible.

El trabajo de la Red se organizó en varias etapas en las que el trabajo colectivo avanzó en ritmo sostenido. La dinámica de la Red se basó en la interacción colaborativa y en la complementariedad de aportaciones en torno al proyecto de investigación común. La investigación comprendió la definición de dimensiones de análisis, referentes empíricos y estrategias metodológicas para la producción de conocimientos específicos en Red que constituyeron una matriz de análisis elaborada colectivamente, un insumo que puede potenciar la realización de futuros estudios comparados.

\section{Principales hallazgos}

A continuación se expone parte los resultados de la investigación a partir de la relectura de los datos sistematizados en cada una de las dimensiones de análisis consideradas. Se sintetiza una selección de los rasgos generales del nivel de posgrado, los mecanismos de regulación más significativos, los sistemas de evaluación y acreditación, las modalidades de financiamiento y los procesos de internacionalización desde una perspectiva comparada en Argentina, Brasil y Paraguay.

\section{Rasgos generales del posgrado en los tres países}

El desarrollo de los posgrados muestra asimetrías y avances institucionales desiguales en Argentina, Brasil y Paraguay así como en cada uno de ellos con puntos de partida y ritmos de crecimiento distintos. En primer lugar cabe señalar la diferencia entre Brasil, por un lado y Argentina y Paraguay, por el otro: en el primero, producto de la colonización portuguesa, fueron creadas instituciones de educación superior recién a comienzos del siglo XIX; en los otros dos, de colonización española fueron establecidas universidades desde el siglo XVI (Soares, 2002, citado por Rodrigues Filho \& Aguilar, 2016). No obstante, si bien Brasil cuenta con su primera universidad, la Universidad de Río de Janeiro, recién en 1920 con un modelo de organización por facultades, desde el inicio fuertemente criticado por su dedicación predominante a la enseñanza, la tensión 
entre una institución universitaria orientada hacia la docencia o hacia la investigación, evolucionó hacia un modelo sostenido en la articulación entre docencia, investigación y extensión, realidad que impactó en el desarrollo temprano de carreras de posgrado. En Argentina el modelo napoleónico derivó en una universidad con dificultades para promover la investigación científica y tecnológica y con ello la formación avanzada. Las actividades de posgrado se promueven en los años sesenta y, como el conjunto de las universidades argentinas de gestión pública, estuvieron sujetas a los vaivenes de la política nacional producto de la intervención durante los regímenes dictatoriales. En la década de 1990, luego de la recuperación democrática del período 1983-1989, en el marco de un gobierno de corte neoliberal se produjo una expansión del posgrado calificada como "explosiva y desordenada" (Barsky \& Dávila, 2004) o "rápida" y "anómica" (Krotsch, 1996) que contribuyó a la configuración de un mapa universitario complejo como resultado de una proliferación no planificada y regulada por el mercado. Como sucedió en la Argentina, en Paraguay según lo expresado por Rivarola "el largo ciclo autoritario que sobrellevó el Paraguay causó un ostensible rezago en su desarrollo económico, político, social y cultural en comparación con el nivel alcanzado por los demás países del Continente" (Rivarola, 2003, p. 71), hecho que afectó a la universidad paraguaya organizada según el modelo de la universidad europea. Recién a partir de 1989 se inicia el proceso de reconstrucción democrática luego de 35 años de dictadura, generando expectativas de cambio en la población que se traducen en la explosión de la matrícula dando lugar a la creación de nuevas universidades, fundamentalmente de gestión privada que absorbieron la mayor parte de la matrícula (Araujo, Balduzzi, Corrado \& Walker, 2016).

En los tres países, entonces, durante la década de 1990 la diversificación institucional producida en un contexto de crecimiento de las instituciones universitarias privadas fue acompañada del desarrollo del nivel cuaternario. Este escenario obedeció al progreso social seguido por las fuerzas productivas, la ciencia y la tecnología que ha conducido a la prolongación de los estudios en las diferentes profesiones (Rama Vitale, 2008). No obstante, la relación entre modernización, investigación, acceso a financiamiento adicional y posgraduación dio lugar a la diferenciación vertical de los sistemas de educación superior con la incorporación de nuevos espacios de trabajo y una creciente complejización por diferenciación de funciones (Araujo, 2017). Por un lado, las exigencias de las instituciones de educación superior, fundamentalmente de las universidades que comenzaron a requerir mayores titulaciones a sus docentes, principalmente de maestría y doctorado, para progresar en la carrera académica. Por el otro, en la mayoría de los países el posgrado se convirtió en una fuente alternativa al financiamiento gubernamental en el marco de la crisis del Estado de bienestar. En países como la Argentina, donde rige la gratuidad para los estudios de grado, el nivel cuaternario así como otras alternativas de posgraduación reguladas por las propias instituciones forman parte del segmento privatizado de la educación dentro de las universidades de gestión estatal. En este sentido, 
es uno de los ámbitos donde se tornaron borrosos los límites entre educación pública y educación privada desde el punto de vista del financiamiento. En algunas instituciones y carreras configuran la expresión de la mercantilización de la educación por cuanto en este nivel la educación deja de ser un derecho o bien público para convertirse en un servicio comercial (Araujo, 2017a).

Si bien el crecimiento es una característica común de los tres países se observan diferencias significativas en la configuración del nivel en cada uno. En la Argentina en 2014 se registraban 2.668 carreras de posgrado concentradas en especializaciones (50.6\%), seguidas de las maestrías (34 \%) y los doctorados (15.4\%). En Brasil la diferenciación entre posgrados lato sensu (especializaciones) y stricto sensu (maestría y doctorado), ha dado origen a un mayor seguimiento y sistematización de información confiable sobre estos últimos regulados por la agencia evaluadora, la Coordinación de Perfeccionamiento del Personal de Educación Superior (CAPES). Si bien se asiste a una mayor regulación de las especializaciones en un contexto de proliferación y diversificación de carreras aún resulta difícil alcanzar un panorama cierto sobre esta realidad. Como contrapartida, los datos sobre el posgrado stricto sensu arrojan la siguiente composición para el año 2014: 3.678 programas de posgrado de los cuales 1.199 (32,6\%) corresponden a maestrías académicas, $1896(51.5 \%)$ a maestrías profesionales, 58 (1,6\%) a doctorados y 525 $(14,3 \%)$ a programas de maestría/doctorado (García, Cristaldo de Benítez, Di Marco, Rodríguez Filho, Zelaya, 2016). En Paraguay se verifica una escasa sistematización de datos sobre la educación superior universitaria en el país, lo cual dificulta el acceso a información con relevancia para el desarrollo de estudios comparados. No obstante, el relevamiento realizado a partir de una muestra de universidades en el marco de esta investigación arrojó para el año 2015 un total de 818 carreras de posgrado, concentradas mayormente en las universidades privadas (623). Se observa la preponderancia de las especializaciones que representan casi la mitad de las carreras (49\%), seguido de las maestrías (40 \%) y por las carreras de doctorado en una proporción mucho menor (11 \%). Un rasgo común en los tres países es la concentración de carreras de orientación profesional, pues predominan las especializaciones y maestrías -con una tendencia al reconocimiento y diferenciación de las maestrías académicas y profesionales. Los doctorados concentran una proporción mucho menor en el escenario de cada país.

El análisis cuantitativo anterior debe ser complementado con otra particularidad, la distribución de los posgrados según regiones, ya que el análisis de los datos evidencia una concentración en las zonas más pobladas y con mayor desarrollo socioeconómico y cultural, como es la región metropolitana y bonaerense en la Argentina, la región Sudeste en Brasil y el Departamento Central en Paraguay. 


\section{Mecanismos actuales de regulación de los posgrados}

El análisis comparado permite dar cuenta del incremento paulatino de normativas específicas para la regulación de los posgrados al compás de la complejización del nivel cuaternario. La creación de carreras con bajas exigencias académicas destinadas a brindar una formación rápida se tradujo en una realidad heterogénea, en el marco de la cual no era posible establecer distinciones entre propuestas que, aunque denominadas de posgrado por cursarse a continuación de carreras de grado, tenían profundas diferencias. Así es que las intervenciones gubernamentales en cada país tuvieron como objetivo consensuar parámetros comunes para el reconocimiento de estudios de posgrado. Diversas normativas han intentado establecer un ordenamiento específico y, entre éstas, las vinculadas con los procesos de acreditación, han jugado un rol central legitimando y jerarquizando propuestas a través del reconocimiento oficial de las titulaciones.

Una regulación primordial es la que delimita qué se considera "estudio, carrera o curso de posgrado" en cada país. En Argentina, Brasil y Paraguay se observa una estructura del posgrado semejante que reconoce carreras de especialización, maestría (académica y profesional) y doctorado y, en el caso de Paraguay, se distingue el doctorado académico o de investigación del profesional. No obstante, en Brasil las especializaciones (posgrado lato sensu) no confieren título sino un certificado de conclusión de estudios y pueden ser ofrecidas libremente por instituciones de educación superior acreditadas y que ofrezcan carreras de grado reconocidas en el área. La regulación, supervisión y evaluación están a cargo de la SERES (Secretaría de Regulación y Supervisión de la Educación Superior) mientras que el posgrados stricto sensu, maestrías y doctorados, son responsabilidad de una agencia de evaluación, la CAPES, con una larga tradición en la educación superior de Brasil. Paraguay también regula las capacitaciones cuyo propósito es brindar una permanente actualización profesional o de investigación a través de propuestas con una carga horaria menor.

Con respecto a las articulaciones entre los tipos de carreras, mientras que en Argentina se puede acceder a una carrera de posgrado con titulaciones obtenidas en instituciones universitarias o instituciones de educación superior no universitaria a cualquier carrera de posgrado reconocida, en Brasil y Paraguay se requiere una maestría para continuar estudios de doctorado.

\section{Sistemas de Evaluación y Acreditación}

La creación de agencias de evaluación y acreditación tuvo su origen en diferentes motivaciones en los países estudiados observándose también disimilitudes en cuanto a su composición, sus funciones e impacto en el sistema. En Brasil la actual CAPES tuvo como antecedente otro organismo creado en la década de 1950 cuyo propósito fue asegurar la presencia de personal especializado para dar respuesta a los emprendimientos públicos y privados para el desarrollo del país. Luego de una serie de cambios 
en su vinculación con organismos de investigación y de regulación del posgrado, la CAPES adoptó un modelo propio de promoción y financiamiento de los posgrados en Brasil, siendo una de sus líneas de acción la evaluación del posgrado stricto sensu. En la Argentina la Comisión Nacional de Evaluación y Acreditación Universitaria (CONEAU) fue creada por la Ley № 24.521/95 en un contexto caracterizado por la proliferación de instituciones de educación superior privadas y una expansión, como se dijo, "explosiva", "desordenada", "rápida" y "anómica" de carreras de posgrado. En Paraguay la necesidad de creación de una agencia evaluadora fue producto de su participación en la acreditación de carreras de grado, MEXA (Mecanismo Experimental de Acreditación para el Reconocimiento de Títulos de Grado), aprobado en 2002 por los Ministros de Educación de los países del Mercosur y de Bolivia y Chile para la acreditación de las carreras de Medicina, Agronomía e Ingeniería. Así, en Paraguay se crea la Agencia Nacional de Evaluación y Acreditación de la Educación Superior (ANEAES) con la sanción de la Ley $N^{\circ} 2.072 / 03$.

En Brasil la CAPES acredita el posgrado stricto sensu, esto es, maestrías académicas y profesionales y doctorados en base a un proceso definido a partir de procedimientos de evaluación externa estandarizados para cada área de saber. La CONEAU en Argentina acredita carreras de especialización, maestría académica y profesional, y doctorado en el marco del artículo 39 de la Ley de Educación Superior N²4.521/95 y de la Resolución N 160/11. En Paraguay la ANEAES tiene como funciones "acreditar la calidad académica de las carreras y programas de post-grado que hubiesen sido objeto de evaluaciones externas por la misma Agencia" y "dar difusión pública oportuna sobre las carreras acreditadas" (Ley N²072/03).

En Argentina y Brasil la acreditación introduce un sistema de jerarquías que sólo en el último se relaciona con el acceso a financiamiento. En Argentina los posgrados pueden ser considerados "A" (Excelente), "B" (Muy bueno) y "C" (Bueno) según el cumplimiento de determinados criterios y estándares predeterminados. En Brasil el proceso de evaluación culmina con un informe que otorga un concepto al programa que se pondera de 1 a 7. Aquellos posgrados que obtienen una calificación igual o superior a 3 conservan el reconocimiento del Ministerio de Educación, mientras que en los conceptos 6 y 7, los posgrados adquieren el estatus de recomendados. Además del reconocimiento oficial del título, las calificaciones inciden directamente en el acceso a prestigio y financiamiento diferenciado.

También el impacto de las agencias es diferente. En el año 2012, la CAPES había evaluado 3.342 programas de posgrado stricto sensu mientras que en 2014 el número ascendía, como se señaló, a 3.678. Hasta el año 2016, desde el inicio de sus actividades, la CONEAU había evaluado 7.671 trámites correspondientes a 4.721 carreras en funcionamiento y 2.950 carreras nuevas. Según datos de 2014, fueron acreditados por el organismo 2.468 posgrados de instituciones públicas y privadas, de los cuales 1.258 
corresponden a Especializaciones, 816 a Maestrías y 394 a Doctorados (CONEAU, 2014). Esta situación contrasta con las acciones de la agencia paraguaya que sólo ha evaluado cinco carreras de Especialización en Pediatría Clínica de cuatro universidades (Universidad Católica Nuestra Señora de la Asunción-sedes Guairá y Asunción-, y Universidades Nacionales de Asunción, del Este y de Itapúa).

\section{Modalidades de Financiamiento}

El relevamiento realizado y las fuentes documentales dieron cuenta de una variedad de esquemas de financiamiento del posgrado, identificándose diferencias según tipo de posgrado y sector (Salto, 2014, Escudero, Salto \& Zalazar, 2016). En Brasil el posgrado en el sector público es subsidiado por el Estado con dos modalidades prevalentes, el pago de los salarios docentes en la modalidad de contrataciones de tiempo completo y sistemas de becas para los alumnos. En Argentina y Paraguay se cobra aranceles a los estudiantes bajo la modalidad de compartir costos, en gran medida para asumir el pago de honorarios docentes, atendiendo con los presupuestos universitarios provenientes del gobierno nacional los gastos corrientes de sostenimiento. En el sector privado, el arancelamiento está desregulado y se rige por las reglas del mercado.

Los tres países cuentan con organismos de fomento de la ciencia y la tecnología que financian alumnos de tiempo completo para cursar doctorados y maestrías, con el requerimiento de ser posgrados acreditados ante las agencias evaluadoras específicas. Entre los financiadores más relevantes podemos mencionar a la CAPES y el Consejo Nacional de Desarrollo Científico y Tecnológico (CNPq) de Brasil, el Consejo Nacional de Investigaciones Científicas y Técnicas (CONICET) y la Agencia Nacional de Promoción Científica y Tecnológica (ANPCyT) de Argentina y el Consejo Nacional de Ciencia y Tecnología (CONACYT) de Paraguay. También se han identificado otras fuentes de financiamiento como empresas del sector público y privado y las propias instituciones, a través de la eximición del pago de aranceles, ayudas económicas y becas. Se registraron disimilitudes en cuanto a los valores de los aranceles en los tres países según el tipo de gestión de las instituciones (público-privado), siendo los aranceles de posgrado en Argentina notoriamente más elevados que en Brasil y Paraguay.

\section{Procesos de Internacionalización de los posgrados}

El estudio ha posibilitado relevar estrategias y mecanismos de internacionalización con diferentes niveles de desarrollo. En Brasil, las iniciativas de internacionalización acompañaron el desarrollo y consolidación de los posgrados desde los años 60 al presente, con fuerte injerencia de agencias gubernamentales como la CAPES, el CNPq y la Fundación para el apoyo a la investigación del Estado de San Pablo (FAPESP), en un modelo que coloca a la internacionalización como una política prioritaria de la educación superior (Morosini, 2009). En Argentina se evidencia un posterior pero creciente interés 
por fortalecer procesos de internacionalización con la definición de políticas a nivel central e institucional mientras que, en Paraguay, las acciones emprendidas y sus resultados parecen depender casi exclusivamente de iniciativas institucionales o individuales.

Las principales modalidades que asume la internacionalización son las movilidades estudiantiles y de profesores, los proyectos de investigación conjuntos, las Redes de investigadores, los posgrados asociados y, más recientemente, los programas académicos de doble titulación.

Un párrafo aparte merecen las acciones de promoción y fortalecimiento de los posgrados con sentido regional, desarrolladas en la última década en el marco del Sector Educativo del MERCOSUR como espacio regional de coordinación de políticas educativas, en línea con los Planes Estratégicos para el sector.

Durante el estudio han podido identificarse un conjunto de dificultades comunes de los procesos de internacionalización, especialmente para la institucionalización de la gestión y coordinación de las acciones, la disponibilidad de información, el reconocimiento académico de las acciones y su financiamiento.

\section{Consideraciones finales}

Como se ha señalado, el desarrollo del posgrado en Argentina, Brasil y Paraguay ha sido sostenido aunque con puntos de partida y ritmos de crecimiento diferentes. Si bien las variaciones nacionales son importantes, no alcanzan para contrarrestar la fuerza regulatoria de la evaluación en la configuración de una oferta cada vez más orientada al mercado. Más allá de las divergencias observadas en cuanto a la estructuración y a la historia y tradiciones de los sistemas de educación superior, todos ellos confluyen en la presencia de sistemas de posgrado organizados en torno a la definición de parámetros de evaluación y acreditación. En este caso la principal política educativa parece ser la política de evaluación que legitima o desacredita propuestas formativas en función del cumplimiento de determinados criterios y estándares nacionales, orientando el desarrollo del nivel.

Si bien el estudio ha podido mostrar una panorámica de conjunto y ha aportado análisis comparativos relevantes en el nivel sistémico, a partir de los logros obtenidos en la primera etapa de trabajo se impone una mirada cualitativa en profundidad sobre las disciplinas e instituciones. Actualmente el estudio se orienta sobre la articulación entre las políticas públicas, las políticas institucionales y las dinámicas institucionales que se generan alrededor de carreras de posgrado en áreas disciplinares similares insertas en las instituciones participantes del proyecto.

Cabe destacar la experiencia de trabajo conjunto entre investigadores de varios países y tradiciones investigativas durante los años de funcionamiento de la Red dado que paulatinamente se ha consolidado un valioso espacio colectivo de producción de conocimientos, 
apoyado en condiciones políticas, académicas e institucionales posibilitadoras de procesos de cooperación académica e integración regional. Se han realizado de manera sinérgica un conjunto de aprendizajes individuales y colectivos en los distintos momentos de conformación, organización y consolidación de la Red, cuya profundización es posible gracias a la continuidad lograda a partir de la renovación del financiamiento.

\section{Referencias Bibliográficas}

Aguilar, L. E. (2011). A pesquisa da política pública educacional a partir da análise satisfactória. Tese de Livre Docência. São Paulo: Universidade Estadual de Campinas.

Aguilar, L. E. (2016). Consideraciones sobre el diseño metodológico para el análisis comparativo. In Lamfri, N. (Coord.) Los posgrados en Argentina, Brasil y Paraguay. Aproximaciones comparadas en contextos de evaluación la calidad de la Educación Superior (pp. 7-20). Córdoba: Encuentro Grupo Editor.

Araujo, S. (2017). La acreditación del posgrado en los países del MERCOSUR. Aproximaciones comparativas. In III Jornadas Nacionales de Investigación en Educación Superior. I Jornadas Regionales de Investigación en Educación Superior. "A cien años de la Reforma de Córdoba". Organizadoras UDELAR, Administración Nacional de Educación Pública, NEIES, Mercosur Educativo, Mercosur. Montevideo, Universidad de la República.

Araujo, S. (2017a). Posgrado, evaluación y acreditación. Convergencias y divergencias en modelos de evaluación latinoamericanos. In VIII Encuentro Nacional y V Latinoamericano La universidad como objeto de investigación (pp. 147-194). Santa Fe: Universidad Nacional del Litoral.

Araujo, S., Balduzzi, M., Corrado, R. \& Walker, V. (2016). Evaluación y acreditación de los posgrados en Argentina, Brasil y Paraguay. Aproximaciones comparativas. In Lamfri, N. (Coord.) Los posgrados en Argentina, Brasil y Paraguay. Aproximaciones comparadas en contextos de evaluación la calidad de la Educación Superior (pp. 147-194). Córdoba: Encuentro Grupo Editor.

Barsky, O. \& Dávila, M. (2004). Las carreras de posgrado en Argentina. In Barsky, O., Sigal, V. \& Dávila, M. (Coords.) Los desafíos de la universidad argentina (pp. 449-485). Buenos Aires: Siglo XXI Editores de Argentina.

Escudero, M.C., Salto, D. \& Zalazar, R. (2016). El financiamiento de los posgrados en Argentina, Brasil y Paraguay en perspectiva internacional y comparada. In Lamfri, N. (Coord.) Los posgrados en Argentina, Brasil y Paraguay. Aproximaciones comparadas en contextos de evaluación la calidad de la Educación Superior (pp. 195-213). Córdoba: Encuentro Grupo Editor.

García, L., Cristaldo de Benítez, M., Di Marco, C., Rodríguez Filho, J. A., \& Zelaya, M. (2016). Situación actual del posgrado en Argentina, Brasil y Paraguay: carreras, estudiantes y egresados. In Lamfri, N. (Coord.) Los posgrados en Argentina, Brasil y Paraguay. Aproximaciones comparadas en contextos de evaluación la calidad de la Educación Superior (pp. 75-145). Córdoba: Encuentro Grupo Editor.

Krotsch, P. (1996). El posgrado en la Argentina: una historia de discontinuidad y fragmentación. Pensamiento Universitario, 4(5), 43-56.

Morosini, M. (2009). A Pós-graduação no Brasil: formação e desafíos. Revista Argentina de Educación Superior, 1(1), 125-152. 
Rama Vitale, C. (2008). Los Posgrados en América Latina y el Caribe en la sociedad del conocimiento. San Luis: Nueva Editorial Universitaria.

Rivarola, D. (2003) La educación superior universitaria en Paraguay. Asunción: IESALC.

Rodrigues Filho, J. A. \& Aguilar, L. E. (2016). Origem e desenvolvimento da pós-graduação na Argentina, Brasil e Paraguai: ensaio comparativo In Lamfri, N. (Coord.). Los posgrados en Argentina, Brasil y Paraguay: aproximaciones comparadas en contextos de evaluación la calidad de la educación superior (pp. 21-35). Córdoba: Encuentro Grupo Editor.

Salto, D. (2014). Governance through the market and the state: postgraduate education in Argentina. Bordón. Revista de Pedagogía, 66(1), 61-74.

Nora Zoila Lamfri Magister en Investigación Educativa - Mención socioantropológica- y Profesora en Ciencias de la Educación por la Universidad Nacional de Córdoba, Argentina. Profesora e investigadora en el área de las políticas educativas con énfasis en políticas públicas, estudios comparados en educación superior, evaluación, gestión y planeamiento de la educación. CIFFyH - IDH; Facultad de Filosofía y Humanidades; Universidad Nacional de Córdoba. E-mail: nlamfri@hotmail.com nlamfri@ffyh.unc.edu.ar ORCID: http://orcid.org/0000-0001-7336-4508

Sonia Marcela Araujo

Doctora en Filosofía y Ciencias de la Educación. Magíster y Diploma Superior en Ciencias Sociales con mención en Educación. Licenciada y Profesora en Ciencias de la Educación. Profesora Titular por concurso en el área Didáctica del Departamento de Educación e Investigadora del Núcleo de Estudios Educacionales y Sociales de la Universidad Nacional del Centro de la Provincia de Buenos Aires, Argentina, en temáticas referidas a formación docente, currículum y evaluación en el campo de la educación superior universitaria.

E-mail: saraujo@fch.unicen.edu.ar ORCID: http://orcid.org/0000-0002-7692-1821

Correspondência Nora Zoila Lamfri Universidad Nacional de Córdoba Av. Haya de la Torrre $s / n$ Pabellón Argentina - Ciudad Universitaria Código postal X5000 HUA

Data de submissão: Novembro 2017

Data de avaliação: Março 2018

Data de publicação: Setembro 2018 